\title{
The APPLE Score - A Novel Score for the Prediction of Rhythm Outcomes after Repeat Catheter Ablation of Atrial Fibrillation
}

\author{
Jelena Kornej* , Gerhard Hindricks, Arash Arya, Philipp Sommer, Daniela Husser, \\ Andreas Bollmann \\ Department of Electrophysiology, Heart Center, Leipzig, Germany \\ * jelena.kornej@gmx.de
}

\section{Abstract}

\section{Background}

Arrhythmia recurrences after catheter ablation occur in up to $50 \%$ within one year but their prediction remains challenging. Recently, we developed a novel score for the prediction of rhythm outcomes after single AF ablation demonstrating superiority to other scores. The current study was performed to 1) prove the predictive value of the APPLE score in patients undergoing repeat $A F$ ablation and 2) compare it with the $\mathrm{CHADS}_{2}$ and $\mathrm{CHA}_{2} \mathrm{DS}_{2}-\mathrm{VASc}$ scores.

Citation: Kornej J, Hindricks G, Arya A, Sommer P, Husser D, Bollmann A (2017) The APPLE Score A Novel Score for the Prediction of Rhythm Outcomes after Repeat Catheter Ablation of Atrial Fibrillation. PLoS ONE 12(1): e0169933. doi:10.1371/journal.pone.0169933

Editor: Elena Cavarretta, Universita degli Studi di Roma La Sapienza, ITALY

Received: September 20, 2016

Accepted: December 22, 2016

Published: January 13, 2017

Copyright: @ 2017 Kornej et al. This is an open access article distributed under the terms of the Creative Commons Attribution License, which permits unrestricted use, distribution, and reproduction in any medium, provided the original author and source are credited.

Data Availability Statement: All relevant data are within the paper.

Funding: The authors received no specific funding for this work.

Competing Interests: The authors have declared that no competing interests exist.

\section{Methods}

Rhythm outcome between 3-12 months after AF ablation were documented. The APPLE score (one point for Age $>65$ years, Persistent AF, imPaired eGFR $\left(<60 \mathrm{ml} / \mathrm{min} / 1.73 \mathrm{~m}^{2}\right)$, LA diameter $\geq 43 \mathrm{~mm}, \mathrm{EF}<50 \%$ ) was calculated in every patient before procedure.

\section{Results}

379 consecutive patients from The Leipzig Heart Center AF Ablation Registry ( $60 \pm 10$ years, $65 \%$ male, $70 \%$ paroxysmal $\mathrm{AF}$ ) undergoing repeat $\mathrm{AF}$ catheter ablation were included. Arrhythmia recurrences were observed in 133 patients (35\%). While the $\mathrm{CHADS}_{2}$ (AUC 0.577, $\mathrm{p}=0.037$ ) and $\mathrm{CHA}_{2} \mathrm{DS}_{2}$-VASc scores (AUC 0.590, $\mathrm{p}=0.015$ ) demonstrated low predictive value, the APPLE score showed better prediction of arrhythmia recurrences (AUC 0.617, $p=0.002$ ) than other scores (both $p<0.001$ ). Compared to patients with an APPLE score of 0 , the risk $(\mathrm{OR})$ for arrhythmia recurrences was 2.9, 3.0 and 6.0 (all $p<0.01$ ) for APPLE scores 1,2 , or $\geq 3$, respectively.

\section{Conclusions}

The novel APPLE score is superior to the $\mathrm{CHADS}_{2}$ and $\mathrm{CHA}_{2} \mathrm{DS}_{2}-\mathrm{VASc}$ scores for prediction of rhythm outcomes after repeat AF catheter ablation. It may be helpful to identify patients with low, intermediate or high risk for recurrences after repeat procedure. 


\section{Introduction}

Atrial fibrillation (AF) is the most common clinical arrhythmia associated with significant complications and impaired quality of life. Although medical therapy has limited efficacy compared to invasive AF treatment, arrhythmia recurrences occur in up to $50 \%$ of patients within one year after first catheter ablation, while after repeat ablation they still occur in up to $20 \%$ [1]. Several observational studies have investigated predictors of arrhythmia recurrence in first procedures. This led to the development of different, partly complicated, scores-e.g. ALAR$\mathrm{MEc}$ and $\mathrm{BASE}-\mathrm{AF}_{2}$-for the prediction of rhythm outcomes following catheter ablation $[2,3]$. Some recent studies analyzed predictive value of widely used $\mathrm{CHA}_{2} \mathrm{DS}_{2}$-VASc score for prediction of arrhythmia recurrences after repeat catheter ablation and demonstrated inconsistent results $[4,5]$.

Based on the results of a previous study [6], we developed and validated a new scoring system for arrhythmia recurrences, i.e. APPLE score, and demonstrated good prediction of arrhythmia recurrences before first ablation [7]. However, whether the APPLE score is useful in prediction of rhythm outcomes in patients following repeat catheter ablation is unknown and was analyzed in this study.

\section{Methods}

The study population consisted of patients from The Heart Center Leipzig AF Ablation Registry, Germany undergoing repeat $(\geq 2)$ ablation according to current guidelines between January 2007 and December 2011. The study was performed according to the Declaration of Helsinki and Institutional Guidelines. Institutional Review Board of Heart Center Leipzig approved the analysis. Patients provided written informed consent. All methods were performed in accordance with the relevant guidelines and regulations.

The APPLE score comprised maximum 5 points (one point for Age $>65$ years, Persistent AF, imPaired eGFR $\left[<60 \mathrm{ml} / \mathrm{min} / 1.73 \mathrm{~m}^{2}\right]$, Left atrial diameter $\geq 43 \mathrm{~mm}$, left ventricular Ejection fraction $<50 \%$, range from 0 to 5 ) and was assessed before procedure. The APPLE score, which is based on clinical variables, is a simple tool with good predictive value and was validated in an external validation set showing similar predictive ability [7].

Mapping and ablation was performed using Ensite NavX, Ensite Velocity (St. Jude Medical, St. Paul, MN, USA) or CARTO 3 (Biosense Webster, Diamond Bar, CA, USA). Trans-septal access and catheter navigation were performed with a steerable sheath (Agilis, St. Jude Medical., St. Paul, MN, USA). A 3D geometry of the LA and the pulmonary veins was obtained and subsequently superimposed on a subtracted 3D-CT or MR-image of the LA. If patients presented with AF, sinus rhythm was restored with electrical cardioversion. In those and in patients presenting with sinus rhythm, completeness of previous antral pulmonary vein isolation and linear lesions was assessed and gaps were closed if necessary. In patients presenting with atrial tachycardia, activation and PPI- mapping and ablation was performed as described previously [8].

After ablation, class I and III antiarrhythmic drugs were not reinitiated and according to the current guidelines [9], oral anticoagulation was prescribed for 3-6 months after catheter ablation and depending on risk stratification of stroke using the $\mathrm{CHADS}_{2}$ or $\mathrm{CHA}_{2} \mathrm{DS}_{2}$-VASc score thereafter [10]. All patients were followed for at least 12 months after catheter ablation and 7-day Holter ECG recordings were performed immediately, 3, 6 and 12 months after the ablation. Additional ECGs and Holter ECG recordings were obtained when patients' symptoms were suggestive of AF. arrhythmia recurrences were defined as any atrial arrhythmia lasting $>30$ seconds between 3 and 12 months after ablation. 


\section{Statistical Analysis}

All statistical analyses were performed with SPSS statistical software version 22 (SPSS Inc., Chicago, USA). Data are presented as means and standard deviation for normally distributed continuous variables and as proportions for categorical variables. The differences between continuous values were assessed using an unpaired two-tailed t-test for normally distributed continuous variables, a Mann-Whitney test for skewed variables, and a chi-square test for nominal variables.

Multivariable logistic regression analysis was performed to identify predictive value of the APPLE, $\mathrm{CHADS}_{2}, \mathrm{CHA}_{2} \mathrm{DS}_{2}$-VASc scores for arrhythmia recurrences. Receiver operating characteristic (ROC) curves were generated for the analysis of $\mathrm{CHADS}_{2}, \mathrm{CHA}_{2} \mathrm{DS}_{2}-\mathrm{VASc}$ and APPLE scores' performance in predicting rhythm outcomes, with the area under the curve being equivalent to the $\mathrm{c}$-index for determining the predictive value for a score.

A two-tailed p-value $<0.05$ was considered as statistically significant. All statistical analyses were performed with SPSS statistical software version 22 .

\section{Results}

Three hundred and seventy nine consecutive patients undergoing repeat AF catheter ablation were included. The clinical characteristics of study population are presented in Table 1.

At the time of re-ablation, there were 194 patients (52\%) in SR, 98 (26\%) with AF and 87 (23\%) with atrial tachycardia's (AT). 57 patients (15\%) had completely isolated pulmonary veins. Of 57 patients presenting to repeat catheter ablation with isolated pulmonary veins, 19 patients (33\%) were in SR, 13 (23\%) had AF and 25 (44\%) AT. Generally, arrhythmia recurrences were observed in 133 patients (35\%). There was no association between arrhythmia recurrences and age, renal impairment, lower EF or LA changes between the first and repeat catheter ablation. Arrhythmia recurrences occurred in $29 \%$ with SR and was $57 \%$ in AF (OR 3.212, 95\% CI 1.957-5.274 versus SR, $\mathrm{p}<0.001$ ), and 40\% in AT (OR 1.620, 95\% CI 0.9672.715 versus $S R, p=0.067)$, respectively $(\mathrm{p}=0.007)$.

Table 1. Clinical characteristics of the study population.

\begin{tabular}{|c|c|c|c|c|}
\hline \multirow[t]{2}{*}{ Variables } & \multirow{2}{*}{$\begin{array}{l}\text { Study population } \\
\qquad \mathbf{n}=\mathbf{3 7 9}\end{array}$} & \multicolumn{2}{|c|}{ Arrhythmia recurrences } & \multirow[b]{2}{*}{$p$-value } \\
\hline & & No $(n=246)$ & Yes $(n=133)$ & \\
\hline Age, years & $60 \pm 10$ & $60 \pm 10$ & $59 \pm 10$ & 0.483 \\
\hline Males, \% & 66 & 33 & 36 & 0.430 \\
\hline Persistent AF, \% & 35 & 29 & 45 & 0.002 \\
\hline $\mathrm{BMI}, \mathrm{kg} / \mathrm{m}^{2}$ & $29 \pm 4.8$ & $28 \pm 4.5$ & $29 \pm 5.2$ & 0.136 \\
\hline eGFR, $\mathrm{ml} / \mathrm{min} / 1.73 \mathrm{~m}^{2}$ & $102 \pm 32$ & $102 \pm 31$ & $102 \pm 35$ & 0.904 \\
\hline Hypertension, \% & 75 & 74 & 77 & 0.526 \\
\hline Diabetes mellitus, $\%$ & 13 & 14 & 12 & 0.738 \\
\hline LA diameter, $\mathrm{mm}$ & $43 \pm 6$ & $42 \pm 6$ & $44 \pm 7$ & 0.005 \\
\hline$\Delta \mathrm{LA}$ diameter, $\mathrm{mm}$ & $0(-4-(+3))$ & $0.5(-4-(+3))$ & $0(-4-(+3))$ & 0.804 \\
\hline$E F, \%$ & $60 \pm 10$ & $60 \pm 9$ & $59 \pm 11$ & 0.601 \\
\hline $\mathrm{CHADS}_{2}$ score & $1.2 \pm 0.9$ & $1.1 \pm 0.9$ & $1.3 \pm 1.0$ & 0.052 \\
\hline $\mathrm{CHA}_{2} \mathrm{DS}_{2}$-VASc score & $2.0 \pm 1.4$ & $2.0 \pm 1.3$ & $2.1 \pm 1.5$ & 0.218 \\
\hline APPLE score & $1.4 \pm 1.0$ & $1.2 \pm 1.0$ & $1.6 \pm 1.0$ & 0.001 \\
\hline
\end{tabular}

Abbreviations: BMI-body mass index, eGFR-estimated glomerular filtration rate, LA-left atrial, $\Delta$ LA-changes in LA diameter before first and repeat procedure, EF-ejection fraction.

doi:10.1371/journal.pone.0169933.t001 
Table 2. Association with arrhythmia recurrences after repeat catheter ablation using different scores.

\begin{tabular}{l|c|c|c}
\hline Scores & OR & $95 \%$ Cl & $P$-value \\
\hline APPLE & 1.422 & $1.155-1.751$ & 0.001 \\
\hline CHADS & 1.243 & $1.005-1.538$ & 0.045 \\
\hline CHA $_{2}$ DS $_{2}$-VASc & 1.094 & $0.948-1.262$ & 0.218 \\
\hline
\end{tabular}

doi:10.1371/journal.pone.0169933.t002

While APPLE (OR 1.422, 95\% CI 1.155-1.751, $\mathrm{p}=0.001)$ and $\mathrm{CHADS}_{2}$ score (OR 1.243, $95 \%$ CI 1.005-1.538, $\mathrm{p}=0.045)$ remained significantly associated with arrhythmia recurrences after repeat catheter ablation, $\mathrm{CHA}_{2} \mathrm{DS}_{2}$-VASc score did not (Table 2). Analyzing prediction of arrhythmia recurrences, both $\mathrm{CHADS}_{2}$ (AUC 0.577, 95\% CI 0.505-0.650, $\mathrm{p}=0.037$ ) and $\mathrm{CHA}_{2} \mathrm{DS}_{2}$-VASc (AUC $0.590,95 \% \mathrm{CI} 0.518-0.663, \mathrm{p}=0.015$ ) scores demonstrated only low predictive value, while the APPLE score ranging from 0 to 5 points, showed significant better prediction (AUC $0.617,95 \%$ CI $0.548-0.687, \mathrm{p}=0.002$ ) compared with other two scores (Fig 1).

Patients with APPLE score of 0 (20\%), 1 (32\%), 2 (23\%), and $\geq 3$ (8\%) had arrhythmia recurrence rates of $18 \%, 38 \%, 39 \%$, and $56 \%$, respectively ( $\mathrm{p}=0.001$, Fig 2 ). Compared to patients with an APPLE score of 0, the risk (OR) for arrhythmia recurrences was 2.9 (95\% CI

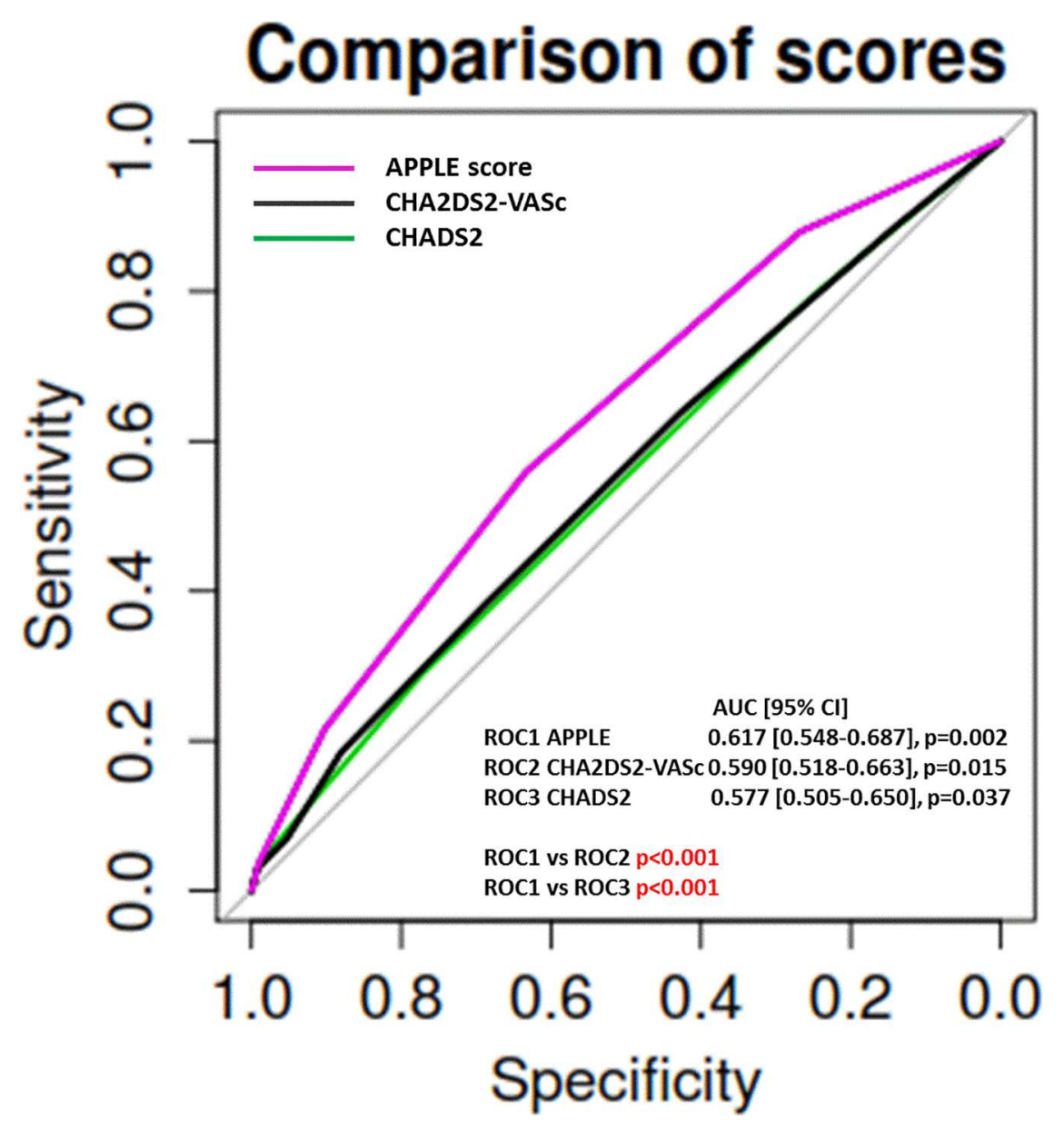

Fig 1. Prediction of arrhythmia recurrences using $\mathrm{CHADS}_{2}, \mathrm{CHA}_{2} \mathrm{DS}_{2}-\mathrm{VASc}$, and APPLE scores. doi:10.1371/journal.pone.0169933.g001 


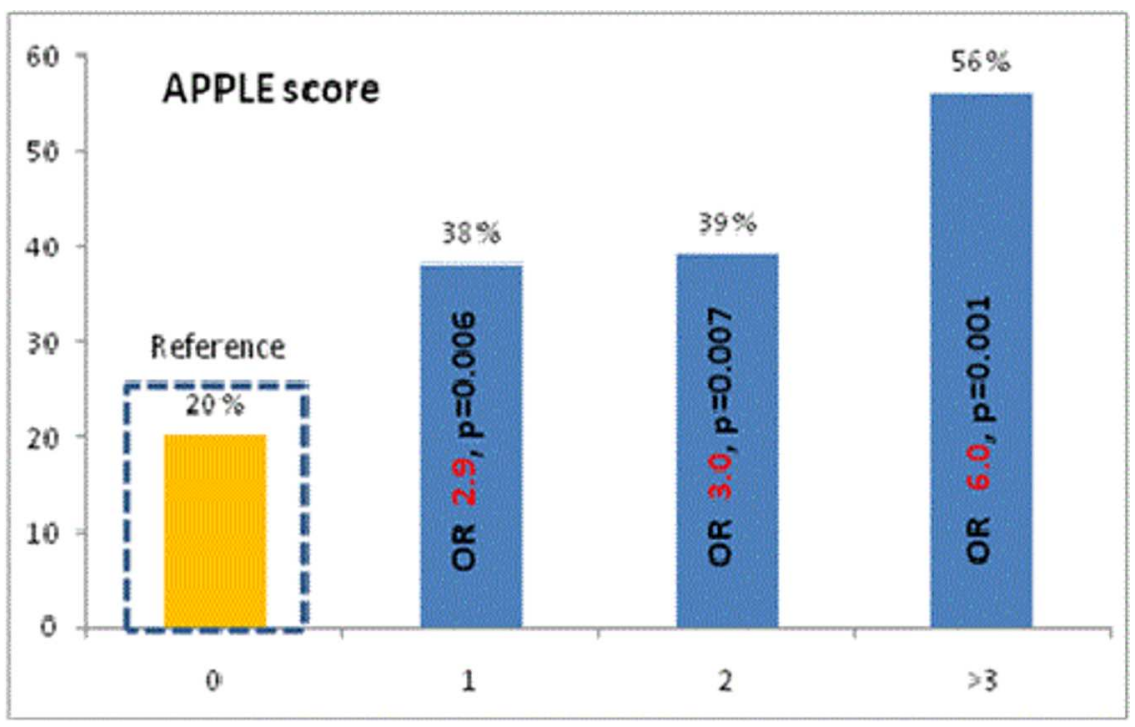

Fig 2. APPLE score and risk for arrythmia recurrences in repeat catheter ablation in whole study population $(\mathbf{n}=\mathbf{3 7 9})$. The figure presents incidence of arrhythmia recurrences $(\%)$ according to each APPLE score point. Compared to patients with an APPLE score of 0 (reference), the risk (OR) for arrhythmia recurrences was $2.9(95 \% \mathrm{Cl} 1.4-6.3, \mathrm{p}=0.006), 3.0(95 \% \mathrm{Cl} 1.3-6.6, \mathrm{p}=0.007)$ and $6.0(95 \% \mathrm{Cl} 2.2-16.8$, $p=0.001$ ) for APPLE scores 1,2 , or $\geq 3$, respectively.

doi:10.1371/journal.pone.0169933.g002

1.4-6.3, $\mathrm{p}=0.006), 3.0(95 \%$ CI 1.3-6.6, $\mathrm{p}=0.007)$ and $6.0(95 \%$ CI $2.2-16.8, \mathrm{p}=0.001)$ for APPLE scores 1,2 , or $\geq 3$, respectively.

\section{Discussion}

\section{Main findings}

In this study, we demonstrate the predictive value of a new scoring system for the prediction of rhythm outcomes after repeat radiofrequency AF catheter ablation in a contemporary AF ablation cohort. Both CHADS2 and APPLE score were significantly associated with arrhythmia recurrences after repeat catheter ablation. However, the APPLE score, which is based on clinical variables, is a novel and simple tool with better predictive value compared to $\mathrm{CHADS}_{2}$ and $\mathrm{CHA}_{2} \mathrm{DS}_{2}$-VASc scores.

\section{APPLE score as predictor for arrhythmia recurrences}

Several studies evaluated the predictive value of different scoring systems that were not specifically designed to predict rhythm outcomes after first AF ablation (e.g. HATCH, $\mathrm{CHADS}_{2}$, $\mathrm{CHA}_{2} \mathrm{DS}_{2}$-VASc scores). A recent large study by Al-Hijji et al [4] failed to demonstrate prediction of arrhythmia recurrences after repeat catheter ablation using $\mathrm{CHA}_{2} \mathrm{DS}_{2}-\mathrm{VASc}$ score, that is in accordance with our results. Nevertheless, in relatively small cohort of patients with longstanding persistent $\mathrm{AF}$ had been recently shown that $\mathrm{CHA}_{2} \mathrm{DS}_{2}$-VASc score $\geq 3$ and renal dysfunction were significantly associated with ablation failure within 31 months [5]. Although, the impact of renal dysfunction on arrhythmia recurrences in patients with first AF ablation had been already shown in our previous research [11], the results of this single center study are difficult to interpret as renal dysfunction (cut off $86 \mathrm{ml} / \mathrm{min}$ ) was not defined in accordance with current KDIGO guidelines [12]. 
Of note, two other scores have been developed to predict rhythm outcomes after invasive AF treatment, as ALARMEc (acronym for AF type, Left Atrium size, Renal insufficiency, MEtabolic syndrome, cardiomyopathy) and BASE- $\mathbf{A F}_{2}$ (acronym for Body mass index $>28 \mathrm{~kg} /$ $\mathrm{m}^{2}$, Atrial dilatation $>40 \mathrm{~mm}$, current Smoking, Early recurrence, AF duration $>6$ years, AF type) $[2,3]$. Both scores were developed using much smaller cohorts of patients undergoing cryoablation compared to our initial cohort [6,7]. Later, Wojcik and co-authors analyzed the predictive value of the ALARMEc score in patients undergoing repeat catheter ablation demonstrating better prediction than $\mathrm{CHADS}_{2}$ and $\mathrm{CHA}_{2} \mathrm{DS}_{2}-\mathrm{VASc}$ scores [3]. Interestingly, between the components of their score, the larger LA size and persistent AF type remained significant predictors for arrhythmia recurrences that was in accordance with our findings, too. Recently, another small study analyzed the predictors for repeat ablation failure in patients with paroxysmal AF and found that changes in LA size was significantly associated with rhythm outcomes [13]. In contrast to these findings, we did not find such association, which might be explained by mixed AF population with both AF types.

\section{Predictors for arrhythmia recurrences}

In a meta-analysis, D'Ascenzo et al [14] demonstrated that persistent AF, LA diameter $>50$ $\mathrm{mm}$ and arrhythmia recurrences within the first month after procedure are the most powerful predictors of AF ablation failure. In contrast to other scores, the APPLE score includes easily obtainable and clearly defined parameters. However, the prediction of arrhythmia recurrences seems to be mostly driven by such components of this score as persistent AF and LA diameter. We did not find significant association between arrhythmia recurrences with age, renal impairment and lower EF. However, it might be explained by 1) small study population, and 2) by young and relatively 'healthy' cohort. Our results are in accordance with previous studies and a recent meta-analysis $[3,6,7,14]$. It seems that the most powerful predictors for the rhythm outcomes remain persistent AF and LA diameter, while other components of APPLE and ALARMEc scores might be considered as possible mediators for arrhythmia occurrence. Nevertheless, using APPLE score it is possible to stratify the risk into different strata that might be helpful for clinical decisions as more aggressive ablation procedure and/or addition of antiarrhythmic drugs within blanking period could be an optimal choice in patients with higher APPLE score. However, the fact that the APPLE score of 0 is still associated with $20 \%$ risk of arrhythmia recurrences in patients after repeat ablation, indicates that this score needs to be further refined.

\section{Arrhythmia recurrences and rhythm type before repeat procedure}

There are different studies analyzing the association between presenting rhythm and ablation outcome. Ammar et al [15] demonstrated that the freedom from any atrial tachyarrhythmia after repeat catheter ablation was reached more often in patients presenting with persistent atrial tachycardia's than in those with recurrent persistent AF, suggesting that atrial tachycardia's might be considered as a step toward sinus rhythm. In accordance to these results, we found that persistent AF type at the time of repeat procedure was significantly associated with adverse rhythm outcomes. Furthermore, not surprisingly patients presenting with persistent AF had higher risk for later arrhythmia recurrences than patients with sinus rhythm.

\section{Limitations}

This study is limited by its observational, retrospective design. Subgroup analysis of recurrence prediction depending on rhythm (AF vs AT) or ablation strategy especially in patients with isolated PVs was not performed due to small sample sizes. Because arrhythmia recurrences 
can be asymptomatic and underdetected, further studies with continuous rhythm monitoring during long-term follow-up are needed to confirm our findings.

\section{Conclusion}

Both CHADS2 and APPLE score were significantly associated with arrhythmia recurrences after repeat catheter ablation. Furthermore, the APPLE score is useful to identify patients with low, intermediate or high risk for arrhythmia recurrences after repeat procedure. Careful evaluation and stratification of patients before repeat procedure using APPLE score may help to identify patients who would profit from repeat invasive AF treatment and improve rhythm outcomes thereafter.

\section{Author Contributions}

Conceptualization: JK AB.

Data curation: JK AB.

Formal analysis: $\mathrm{JK} \mathrm{AB}$.

Funding acquisition: JK GH DH AB.

Investigation: $\mathrm{GH} A \mathrm{AA} P \mathrm{AB}$.

Methodology: JK AB.

Project administration: $\mathrm{GH} A B$.

Resources: JK GH DH AB.

Supervision: $\mathrm{AB}$.

Visualization: JK.

Writing - original draft: JK.

Writing - review \& editing: $\mathrm{JK} \mathrm{AB}$.

\section{References}

1. Ganesan AN, Shipp NJ, Brooks AG, Kuklik P, Lau DH, Lim HS, et al. Long-term outcomes of catheter ablation of atrial fibrillation: a systematic review and meta-analysis. J Am Heart Assoc 2013; 2:e004549. doi: 10.1161/JAHA.112.004549 PMID: 23537812

2. Canpolat U, Aytemir K, Yorgun H, Sahiner L, Kaya EB, Oto A. A proposal for a new scoring system in the prediction of catheter ablation outcomes: Promising results from the Turkish Cryoablation Registry. Int J Cardiol 2013; 169(3):201-206. doi: 10.1016/j.ijcard.2013.08.097 PMID: 24063932

3. Wojcik M, Berkowitsch A, Greiss H, Zaltsberg S, Pajitnev D, Deubner N, et al. Repeated catheter ablation of atrial fibrillation: how to predict outcome? Circ J 2013; 77:2271-2279. PMID: 23759661

4. Al-Hijji MA, Deshmukh AJ, Yao X, Mwangi R, Sangaralingham LR, Friedman PA, et al. Trends and predictors of repeat catheter ablation for atrial fibrillation. Am Heart J. 2016; 171(1):48-55. doi: 10.1016/j. ahj.2015.10.015 PMID: 26699600

5. Wang Q, Jiang SL, Liu X, Yang YQ. Repeat Catheter Ablation of Long-standing Persistent Atrial Fibrillation in Patients with a Total Atrial Fibrillation Duration of More Than 2 Years: Effects of the CHA2DS2VASc Score and Estimated Glomerular Filtration Rate on the Outcomes. Intern Med. 2016; 55 (18):2537-47. doi: 10.2169/internalmedicine.55.5790 PMID: 27629945

6. Kornej J, Hindricks G, Kosiuk J, Arya A, Sommer P, Husser D, et al. Comparison of CHADS2, R2CHADS2 and CHA2DS2-VASc scores for prediction of rhythm outcomes after catheter ablation of atrial fibrillation: the Leipzig Heart Center AF Ablaiton Registry. Circ Arrhythm Electrophysiol 2014; 7:281-287. doi: 10.1161/CIRCEP.113.001182 PMID: 24610790 
7. Kornej J, Hindricks G, Shoemaker MB, Husser D, Arya A, Sommer P, et al. The APPLE score: a novel and simple score for the prediction of rhythm outcomes after catheter ablation of atrial fibrillation. Clin Res Cardiol 2015; 104(10):871-876. doi: 10.1007/s00392-015-0856-x PMID: 25876528

8. Esato M, Hindricks G, Sommer P, Arya A, Gaspar T, Bode K, et al. Color-coded three-dimensional entrainment mapping for analysis and treatment of atrial macroreentrant tachycardia. Heart Rhythm. 2009; 6(3):349-358. doi: 10.1016/j.hrthm.2008.12.013 PMID: 19251210

9. Kirchhof P, Benussi S, Kotecha D, Ahlsson A, Atar D, Casadei B, Castella M, Diener HC, Heidbuchel H, Hendriks J, Hindricks G, Manolis AS, Oldgren J, Popescu BA, Schotten U, Van Putte B, Vardas P. 2016 ESC Guidelines for the management of atrial fibrillation developed in collaboration with EACTS: The Task Force for the management of atrial fibrillation of the European Society of Cardiology (ESC)Developed with the special contribution of the European Heart Rhythm Association (EHRA) of the ESCEndorsed by the European Stroke Organisation (ESO). Eur Heart J. 2016 [Epub ahead of print]

10. Lip GY, Nieuwlaat R, Pisters R, Lane DA, Crijns HJ. Refining clinical risk stratification for predicting stroke and thromboembolism in atrial fibrillation using a novel risk factor-based approach: the euro heart survey on atrial fibrillation. Chest 2010; 137:263-272. doi: 10.1378/chest.09-1584 PMID: 19762550

11. Kornej J, Hindricks G, Banerjee A, Arya A, Sommer P, Rolf S, Husser D, Lip GY, Bollmann A. Changes in renal function after catheter ablation of atrial fibrillation are associated with CHADS2 and CHA2DS2VASc scores and arrhythmia recurrences. Heart. 2015; 101(2):126-31. doi: 10.1136/heartjnl-2014306013 PMID: 25281600

12. Levey AS, de Jong PE, Coresh J, EI Nahas M, Astor BC, Matsushita K, Gansevoort RT, Kasiske BL, Eckardt KU. The definition, classification, and prognosis of chronic kidney disease: a KDIGO Controversies Conference report. Kidney Int. 2011; 80(1):17-28. doi: 10.1038/ki.2010.483 PMID: 21150873

13. Tang RB, Yan XL, Dong JZ, Kalifa J, Long DY, Yu RH, et al. Predictors of recurrence after a repeat ablation procedure for paroxysmal atrial fibrillation: role of left atrial enlargement. Europace 2014; 16:15691574. doi: 10.1093/europace/euu013 PMID: 24692517

14. D'Ascenzo F, Corleto A, Biondi-Zoccai G, Anselmino M, Ferraris F, di Biase L, et al. Which are the most reliable predictors of recurrence of atrial fibrillation after transcatheter ablation? A meta-analysis. Int $J$ Cardiol. 2013; 167:1984-9. doi: 10.1016/j.ijcard.2012.05.008 PMID: 22626840

15. Ammar S, Hessling G, Reents T, Fichtner S, Wu J, Zhu P et al. Arrhythmia type after persistent atrial fibrillation ablation predicts success of the repeat procedure. Circ Arrhythm Electrophysiol 2011; 4:60914. doi: 10.1161/CIRCEP.111.963256 PMID: 21856772 